TPR-96-08 (revised); hep-th/9606055

\title{
Three-Point Functions at Finite Temperature
}

\author{
M.E. Carringtont and U. Heinz \\ Institut für Theoretische Physik, Universität Regensburg, \\ D-93040 Regensburg, Germany
}

(April 20, 2018)

\begin{abstract}
We study 3-point functions at finite temperature in the closed time path formalism. We give a general decomposition of the eight component tensor in terms of seven vertex functions. We derive a spectral representation for these seven functions in terms of two independent real spectral functions. We derive relationships between the seven functions and obtain a representation of the vertex tensor that greatly simplifies calculations in real time.
\end{abstract}

PACS numbers: $11.10 \mathrm{Wx}, 11.15 \mathrm{Tk}, 11.55 \mathrm{Fv}$

Typeset using REVTEX 


\section{INTRODUCTION}

In this paper, we discuss the analytical structure of the three-point function at finite temperature. We consider bosonic fields and work in the real time formalism. Throughout this paper we use the Keldysh or closed time path (CTP) formalism [1 [3]. The integration contour has one branch running from negative infinity to positive infinity, and a second branch running backwards from positive infinity to negative infinity. We call the top branch of this contour $C_{1}$ and the bottom branch $C_{2}$. The arguments of the fields can take values on either branch of this contour. This choice of contour then leads to a doubling of degrees of freedom.

Historically, the real time formalism has been much less popular than the imaginary time formalism because of the mathematical difficulties associated with the doubling of degrees of freedom. However, in some cases calculations in the real time formalism are much simpler. In particular, the real time formalism is extremely useful when working with ultra soft external energy scales. Examination of the pole structure of the internal propagators allows one to identify infinite sets of graphs that contribute to the same order in perturbation theory. For example, in a theory with an interaction of the form $g \phi^{3}$, the ladder graphs contribute terms to the self energy of order $g^{2 L} T^{1+L} / k^{L-1}$ when the number of loops $L$ is larger than 1 [4. 5 . When the pole structure of all propagators is explicit it is easy to extract the piece of each graph that dominates when $k \sim g^{2} T$. In order to obtain complete results, all such contributions must be resummed. This requires an efficient and economic set of calculational tools for real-time perturbation theory to which we wish to contribute with the present paper.

The analytic structure of the three-point vertex at finite temperature has been studied previously in several publications, both in imaginary time [6,7] and real time [8 [12. The real time formulation has the advantage that it avoids the need for analytic continuation to real frequencies which, for higher order $n$-point functions, can easily become a source of confusion. Its disadvantage is the occurrence of many different thermal components of the $n$-point 
functions which results from the doubling of degrees of freedom. The resulting proliferation of terms in real-time calculations has contributed considerably to the lack of popularity of the real-time formalism. In the actual calculations one finds, however, that a large number of terms cancel in the end. The vertex representations found in the previous papers [8 12] do not implement these cancellation mechanisms at all, or only incompletely. We derive here a different (though mathematically equivalent) decomposition and spectral representation of the real-time three-point vertex at finite temperature. We demonstrate that it leads to impressive simplifications in perturbative calculations by making the physical cancellations explicit on an algebraic level in the first step of the calculation, thereby dramatically reducing the number of terms which need to be evaluated.

\section{SINGLE-PARTICLE PROPAGATOR}

To establish our notation and for later use we first consider the single-particle propagator. In real time, the propagator has $2^{2}=4$ components, since each of the two fields can take values on either branch of the contour. Thus, the two-point function can be written as a $2 \times 2$ matrix of the form

$$
D=\left(\begin{array}{ll}
D_{11} & D_{12} \\
D_{21} & D_{22}
\end{array}\right),
$$

where $D_{11}$ is the propagator for fields moving along $C_{1}, D_{12}$ is the propagator for fields moving from $C_{1}$ to $C_{2}$, etc. The four components are given by [13]

$$
\begin{aligned}
& D_{11}(x-y)=-i\langle T(\phi(x) \phi(y))\rangle, \\
& D_{12}(x-y)=-i\langle\phi(y) \phi(x)\rangle, \\
& D_{21}(x-y)=-i\langle\phi(x) \phi(y)\rangle, \\
& D_{22}(x-y)=-i\langle\tilde{T}(\phi(x) \phi(y))\rangle,
\end{aligned}
$$

where $T$ is the usual time ordering operator, and $\tilde{T}$ is the antichronological time ordering operator. These four components satisfy 


$$
D_{11}-D_{12}-D_{21}+D_{22}=0
$$

as a consequence of the identity $\theta(x)+\theta(-x)=1$.

It is more useful to write the propagator in terms of the three functions

$$
\begin{aligned}
& D_{R}=D_{11}-D_{12}, \\
& D_{A}=D_{11}-D_{21}, \\
& D_{F}=D_{11}+D_{22} .
\end{aligned}
$$

$D_{R}$ and $D_{A}$ are the usual retarded and advanced propagators satisfying

$$
D_{R}(x-y)-D_{A}(x-y)=-i\langle[\phi(x), \phi(y)]\rangle,
$$

and $D_{F}$ is the symmetric combination

$$
D_{F}(x-y)=-i\langle\{\phi(x), \phi(y)\}\rangle .
$$

In Appendix A1 we show that in momentum space these propagators are related by the well-known fluctuation-dissipation theorem [14]

$$
D_{F}(p)=\left(1+2 n\left(p_{0}\right)\right)\left(D_{R}(p)-D_{A}(p)\right)=-i\left(1+2 n\left(p_{0}\right)\right) \rho_{-}(p) .
$$

where $n\left(p_{0}\right)$ is the thermal Bose-Einstein distribution

$$
n\left(p_{0}\right)=\frac{1}{e^{\beta p_{0}}-1} .
$$

$\rho_{-}(p)$ is the spectral density in terms of which all components of the single-particle propagator can be expressed via spectral integrals, for example

$$
D_{R, A}(p)=\int_{-\infty}^{\infty} \frac{d \omega}{2 \pi} \frac{\rho_{-}(\omega, \mathbf{p})}{p_{0}-\omega \pm i \epsilon} .
$$

Equations (3) and (四) are inverted by

$$
\begin{aligned}
D_{11} & =\frac{1}{2}\left(D_{F}+D_{A}+D_{R}\right), \\
D_{12} & =\frac{1}{2}\left(D_{F}+D_{A}-D_{R}\right), \\
D_{21} & =\frac{1}{2}\left(D_{F}-D_{A}+D_{R}\right), \\
D_{22} & =\frac{1}{2}\left(D_{F}-D_{A}-D_{R}\right) .
\end{aligned}
$$


These equations can be written in a more convenient notation as [15]

$$
2 D=D_{R}\left(\begin{array}{l}
1 \\
1
\end{array}\right)\left(\begin{array}{c}
1 \\
-1
\end{array}\right)+D_{A}\left(\begin{array}{c}
1 \\
-1
\end{array}\right)\left(\begin{array}{l}
1 \\
1
\end{array}\right)+D_{F}\left(\begin{array}{l}
1 \\
1
\end{array}\right)\left(\begin{array}{l}
1 \\
1
\end{array}\right)
$$

where the outer product of the column vectors is to be taken. Using the relation Eq. (đ) we further obtain [16]

$$
D(p)=D_{R}(p)\left(\begin{array}{l}
1 \\
1
\end{array}\right)\left(\begin{array}{c}
1+n\left(p_{0}\right) \\
n\left(p_{0}\right)
\end{array}\right)-D_{A}(p)\left(\begin{array}{c}
n\left(p_{0}\right) \\
1+n\left(p_{0}\right)
\end{array}\right)\left(\begin{array}{l}
1 \\
1
\end{array}\right) .
$$

For later use we give the decomposition of the inverse propagator:

$$
D^{-1}(p)=\frac{1}{D_{R}(p)}\left(\begin{array}{c}
1 \\
-1
\end{array}\right)\left(\begin{array}{c}
1+n\left(p_{0}\right) \\
-n\left(p_{0}\right)
\end{array}\right)+\frac{1}{D_{A}(p)}\left(\begin{array}{c}
-n\left(p_{0}\right) \\
1+n\left(p_{0}\right)
\end{array}\right)\left(\begin{array}{c}
1 \\
-1
\end{array}\right) .
$$

These representations are very useful for doing calculations in real time.

Similar relations can be obtained for the polarization tensor, although they are perturbatively less useful. The polarization tensor is the 1PI two-point function and is obtained by amputating the external legs from the single-particle propagator. The Dyson equation gives

$$
i D(p)=i D_{0}(p)+i D_{0}(p)(-i \Pi(p)) i D(p)
$$

The analogues of Eqs. (3) and (田) are

$$
\begin{aligned}
& \Pi_{R}=\Pi_{11}+\Pi_{12}, \\
& \Pi_{A}=\Pi_{11}+\Pi_{21}, \\
& \Pi_{F}=\Pi_{11}+\Pi_{22},
\end{aligned}
$$

and

$$
\Pi_{11}+\Pi_{12}+\Pi_{21}+\Pi_{22}=0 .
$$

The analogues of Eqs. (11), (71) and (12) are

$$
\begin{aligned}
2 \Pi(p) & =\Pi_{R}(p)\left(\begin{array}{c}
1 \\
-1
\end{array}\right)\left(\begin{array}{l}
1 \\
1
\end{array}\right)+\Pi_{A}(p)\left(\begin{array}{l}
1 \\
1
\end{array}\right)\left(\begin{array}{c}
1 \\
-1
\end{array}\right)+\Pi_{F}(p)\left(\begin{array}{c}
1 \\
-1
\end{array}\right)\left(\begin{array}{c}
1 \\
-1
\end{array}\right), \\
\Pi_{F}(p) & =\left(1+2 n\left(p_{0}\right)\right)\left(\Pi_{R}(p)-\Pi_{A}(p)\right), \\
\Pi(p) & =\Pi_{R}(p)\left(\begin{array}{c}
1 \\
-1
\end{array}\right)\left(\begin{array}{c}
1+n\left(p_{0}\right) \\
-n\left(p_{0}\right)
\end{array}\right)+\Pi_{A}(p)\left(\begin{array}{c}
-n\left(p_{0}\right) \\
1+n\left(p_{0}\right)
\end{array}\right)\left(\begin{array}{c}
1 \\
-1
\end{array}\right) .
\end{aligned}
$$




\section{THREE-POINT FUNCTION}

In the real time formalism, the three-point function has $2^{3}=8$ components. We denote these by $\Gamma_{a b c}$ with $\{a, b, c=1,2\}$ where, for example,

$$
\begin{aligned}
& \Gamma_{111}(x, y, z)=\langle T(\phi(x) \phi(y) \phi(z))\rangle, \\
& \Gamma_{112}(x, y, z)=\langle\phi(z) T(\phi(x) \phi(y))\rangle,
\end{aligned}
$$

etc. The full set of vertex functions can be found in Refs. [15, 13]. Only seven of these eight components are independent because of the identity

$$
\sum_{a, b, c=1}^{2}(-1)^{a+b+c-3} \Gamma_{a b c}=0
$$

which follows in the same way as Eq. (3) from $\theta(x)+\theta(-x)=1$. The seven combinations that we use are defined as [15]

$$
\begin{gathered}
\Gamma_{R}(x, y, z)=\Gamma_{111}-\Gamma_{112}-\Gamma_{211}+\Gamma_{212}, \\
\Gamma_{R i}(x, y, z)=\Gamma_{111}-\Gamma_{112}-\Gamma_{121}+\Gamma_{122}, \\
\Gamma_{R o}(x, y, z)=\Gamma_{111}-\Gamma_{121}-\Gamma_{211}+\Gamma_{221}, \\
\Gamma_{F}(x, y, z)=\Gamma_{111}-\Gamma_{121}+\Gamma_{212}-\Gamma_{222}, \\
\Gamma_{F i}(x, y, z)=\Gamma_{111}+\Gamma_{122}-\Gamma_{211}-\Gamma_{222}, \\
\Gamma_{F o}(x, y, z)=\Gamma_{111}-\Gamma_{112}+\Gamma_{221}-\Gamma_{222}, \\
\Gamma_{E}(x, y, z)=\Gamma_{111}+\Gamma_{122}+\Gamma_{212}+\Gamma_{221}
\end{gathered}
$$

Clearly, the set Eq. (22) contains the same information as Eqs. (20) and (21). It merely defines a change of basis to a different set of independent functions, just as Eq. (雨) defines a change of independent functions for the single-particle propagator.

In coordinate space we always label the first leg of the three-point function by $x$ and call it the "incoming leg $(i)$ ", the third leg we label by $z$ and call it the "outgoing leg $(o)$ ", and the second (middle) leg we label by $y$. Using Eq. (20) we can rewrite Eq. (22) to obtain expressions for the vertices in terms of commutators of the fields. With the obvious 
shorthands $\phi_{1} \equiv \phi(x), \phi_{2} \equiv \phi(y), \phi_{3} \equiv \phi(z)$, and $\theta_{12} \equiv \theta\left(x_{0}-y_{0}\right)$, etc., one thus finds that the first three vertices in Eq. (22) are just the retarded vertex functions:

$$
\begin{gathered}
\Gamma_{R}=\theta_{23} \theta_{31}\left\langle\left[\left[\phi_{2}, \phi_{3}\right], \phi_{1}\right]\right\rangle+\theta_{21} \theta_{13}\left\langle\left[\left[\phi_{2}, \phi_{1}\right], \phi_{3}\right]\right\rangle, \\
\Gamma_{R i}=\theta_{12} \theta_{23}\left\langle\left[\left[\phi_{1}, \phi_{2}\right], \phi_{3}\right]\right\rangle+\theta_{13} \theta_{32}\left\langle\left[\left[\phi_{1}, \phi_{3}\right], \phi_{2}\right]\right\rangle, \\
\Gamma_{R o}=\theta_{32} \theta_{21}\left\langle\left[\left[\phi_{3}, \phi_{2}\right], \phi_{1}\right]\right\rangle+\theta_{31} \theta_{12}\left\langle\left[\left[\phi_{3}, \phi_{1}\right], \phi_{2}\right]\right\rangle .
\end{gathered}
$$

$\Gamma_{R i}$ is the vertex retarded with respect to $x_{0}, \Gamma_{R o}$ is retarded with respect to $z_{0}$, and $\Gamma_{R}$ is

retarded with respect to $y_{0}$. The four remaining vertices contain various symmetric combinations of the fields.

The inversion of Eq. (22) can be elegantly presented in tensor notation in terms of the two-component column vectors introduced [15] in the previous Section:

$$
\begin{aligned}
4 \Gamma & =\Gamma_{R}\left(\begin{array}{c}
1 \\
-1
\end{array}\right)\left(\begin{array}{l}
1 \\
1
\end{array}\right)\left(\begin{array}{c}
1 \\
-1
\end{array}\right)+\Gamma_{R i}\left(\begin{array}{l}
1 \\
1
\end{array}\right)\left(\begin{array}{c}
1 \\
-1
\end{array}\right)\left(\begin{array}{c}
1 \\
-1
\end{array}\right) \\
& +\Gamma_{R o}\left(\begin{array}{c}
1 \\
-1
\end{array}\right)\left(\begin{array}{c}
1 \\
-1
\end{array}\right)\left(\begin{array}{l}
1 \\
1
\end{array}\right)+\Gamma_{F}\left(\begin{array}{l}
1 \\
1
\end{array}\right)\left(\begin{array}{c}
1 \\
-1
\end{array}\right)\left(\begin{array}{l}
1 \\
1
\end{array}\right) \\
& +\Gamma_{F i}\left(\begin{array}{c}
1 \\
-1
\end{array}\right)\left(\begin{array}{l}
1 \\
1
\end{array}\right)\left(\begin{array}{l}
1 \\
1
\end{array}\right)+\Gamma_{F o}\left(\begin{array}{l}
1 \\
1
\end{array}\right)\left(\begin{array}{l}
1 \\
1
\end{array}\right)\left(\begin{array}{c}
1 \\
-1
\end{array}\right) \\
& +\Gamma_{E}\left(\begin{array}{l}
1 \\
1
\end{array}\right)\left(\begin{array}{l}
1 \\
1
\end{array}\right)\left(\begin{array}{l}
1 \\
1
\end{array}\right) .
\end{aligned}
$$

This decomposition of the thermal vertex tensor is analogous to Eq. (11) for the propagator. Its simple structure results from our choice Eq. (22) for the basis functions. In the following Section we show that it is possible to further simplify this decomposition by deriving relationships between the vertex functions (in analogy to Eq. (7)). The usefulness of these expressions for performing explicit calculations is demonstrated in Sec. IV.

\section{A. Spectral representation of the three-point functions}

In Appendix A2 we show in detail that the seven three-point functions Eq. (22) can be rewritten in terms of two independent spectral densities. Starting from (23), the three-point functions are constructed as products of theta functions and expectation values of the form 


$$
\left\langle\phi_{a} \phi_{b} \phi_{c}\right\rangle=\sum_{l}\left\langle l\left|e^{-\beta H} \phi_{a} \phi_{b} \phi_{c}\right| l\right\rangle
$$

We calculate these expectation values in the usual way, by going to the Heisenberg representation and using translation invariance in the form of Eq. (A1), and inserting complete sets of states between the operators. Because of the cyclic property of the trace, there are only two independent expectation values. This allows us to write all of the three-point functions in terms of two spectral functions. We find it useful to define

$$
\begin{aligned}
& \rho_{A}=\rho_{1}+\rho_{3} \\
& \rho_{B}=\rho_{2}+\rho_{4}
\end{aligned}
$$

where

$$
\begin{aligned}
& \rho_{1}(p, k)=(2 \pi)^{8} \sum_{l m n} M_{n m l}\left(e^{-\beta E_{l}}-e^{-\beta E_{m}}\right) \delta\left(p-\frac{1}{2} k+p_{l}-p_{n}\right) \delta\left(p+\frac{1}{2} k+p_{l}-p_{m}\right), \\
& \rho_{2}(p, k)=(2 \pi)^{8} \sum_{l m n} M_{n m l}\left(e^{-\beta E_{n}}-e^{-\beta E_{m}}\right) \delta\left(p-\frac{1}{2} k+p_{l}-p_{n}\right) \delta\left(p+\frac{1}{2} k+p_{l}-p_{m}\right), \\
& \rho_{3}(p, k)=(2 \pi)^{8} \sum_{l m n} M_{n m l}\left(e^{-\beta E_{l}}-e^{-\beta E_{n}}\right) \delta\left(p-\frac{1}{2} k+p_{m}-p_{l}\right) \delta\left(p+\frac{1}{2} k+p_{n}-p_{l}\right), \\
& \rho_{4}(p, k)=(2 \pi)^{8} \sum_{l m n} M_{n m l}\left(e^{-\beta E_{m}}-e^{-\beta E_{n}}\right) \delta\left(p-\frac{1}{2} k+p_{m}-p_{l}\right) \delta\left(p+\frac{1}{2} k+p_{n}-p_{l}\right),
\end{aligned}
$$

with $M_{n m l}=\langle l|\phi(0)| n\rangle\langle n|\phi(0)| m\rangle\langle m|\phi(0)| l\rangle=M_{l m n}^{*}$. The two independent spectral functions $\rho_{A}$ and $\rho_{B}$ are real in coordinate space since

$$
\rho_{A, B}(p, k)=\rho_{A, B}^{*}(-p,-k) .
$$

Another way of verifying that there are only two independent real spectral densities is as follows: all four spectral functions $\rho_{i}(p, k), i=1, \ldots, 4$, in Eq. (27) can be expressed in terms of a single complex function,

$$
\rho(p, k)=(2 \pi)^{8} \sum_{l m n} M_{n m l} e^{-\beta E_{l}} \delta\left(p-\frac{1}{2} k+p_{l}-p_{n}\right) \delta\left(p+\frac{1}{2} k+p_{l}-p_{m}\right),
$$

by expressing all energies in the Boltzmann factors in terms of $E_{l}$, using the $\delta$-functions, and appropriately relabelling the summation indices. So there is really only one complex function (or, equivalently, two real functions) of $p$ and $k$ which contain all the physical information on the (non-perturbative) analytical structure of the vertex. 
We now write down the spectral representation of the vertex functions in momentum space, $\Gamma_{\alpha}\left(p_{1}, p_{2}, p_{3}\right)$, where $p_{1}, p_{2}, p_{3}$ (with $\left.p_{i}=\left(E_{i}, \mathbf{p}_{i}\right)\right)$ are the momenta associated with $x, y, z$, respectively (i.e. the three momenta flowing into the three legs of the vertex). Due to translation invariance $p_{1}+p_{2}+p_{3}=0$, and we exploit this by writing $p_{1}=p-k / 2$, $p_{2}=k$, and $p_{3}=-(p+k / 2)$.

We use the following shorthand notation:

$$
\begin{aligned}
\Gamma_{\alpha} & \equiv \Gamma_{\alpha}(p, k) \equiv \Gamma_{\alpha}(p-k / 2, k,-p-k / 2)=\Gamma_{\alpha}\left(p_{1}, p_{2}, p_{3}\right), \quad \alpha=R, F, E, \ldots ; \\
\rho_{A, B} & \equiv \rho_{A, B}\left(\omega_{1}, \mathbf{p} ; \omega_{2}, \mathbf{k}\right) ; \\
a_{j}^{ \pm} & =\frac{1}{E_{j}-\Omega_{j} \pm i \epsilon}, \quad j=1,2,3 ; \\
\Omega_{1} & =\omega_{1}-\frac{1}{2} \omega_{2}, \quad \Omega_{2}=\omega_{2}, \quad \Omega_{3}=-\omega_{1}-\frac{1}{2} \omega_{2}, \quad \Omega_{1}+\Omega_{2}+\Omega_{3}=0 ; \\
\tilde{N}_{i} & =1+2 n\left(\Omega_{i}\right), \quad i=1,2,3 ; \\
\int & =\int_{-\infty}^{\infty} \frac{d \omega_{1}}{2 \pi} \frac{d \omega_{2}}{2 \pi} .
\end{aligned}
$$

Then the spectral integrals for the vertex functions are

$$
\begin{aligned}
& \Gamma_{R}= \int\left[\left(\rho_{B}-\rho_{A}\right) a_{1}^{-}-\rho_{A} a_{3}^{-}\right] a_{2}^{+} \\
& \Gamma_{R i}= \int\left[\rho_{A} a_{3}^{-}+\rho_{B} a_{2}^{-}\right] a_{1}^{+}, \\
& \Gamma_{R o}= \int\left[\left(\rho_{A}-\rho_{B}\right) a_{1}^{-}-\rho_{B} a_{2}^{-}\right] a_{3}^{+}, \\
& \Gamma_{F}=\int\left[\tilde{N}_{1}\left(\rho_{B}-\rho_{A}\right)\left(a_{1}^{+} a_{2}^{-}+a_{1}^{-} a_{3}^{+}+a_{2}^{-} a_{3}^{+}\right)-\tilde{N}_{3} \rho_{A}\left(a_{1}^{+} a_{2}^{-}+a_{1}^{+} a_{3}^{-}+a_{2}^{-} a_{3}^{+}\right)\right] \\
& \Gamma_{F i}=\int\left[\tilde{N}_{3} \rho_{A}\left(a_{1}^{-} a_{2}^{+}+a_{1}^{-} a_{3}^{+}+a_{2}^{+} a_{3}^{-}\right)+\tilde{N}_{2} \rho_{B}\left(a_{1}^{-} a_{2}^{+}+a_{1}^{-} a_{3}^{+}+a_{2}^{-} a_{3}^{+}\right)\right] \\
& \Gamma_{F o}=\int\left[\tilde{N}_{1}\left(\rho_{A}-\rho_{B}\right)\left(a_{1}^{-} a_{2}^{+}+a_{1}^{+} a_{3}^{-}+a_{2}^{+} a_{3}^{-}\right)-\tilde{N}_{2} \rho_{B}\left(a_{1}^{+} a_{2}^{-}+a_{1}^{+} a_{3}^{-}+a_{2}^{+} a_{3}^{-}\right)\right] \\
& \Gamma_{E}=\int\left[\left(\tilde{N}_{1} \tilde{N}_{3}\left(\rho_{B}-\rho_{A}\right)+\tilde{N}_{2} \tilde{N}_{3} \rho_{B}\right)\left(a_{1}^{+}+a_{2}^{+}\right) a_{3}^{-}-\tilde{N}_{1}\left(\tilde{N}_{3} \rho_{A}+\tilde{N}_{2} \rho_{B}\right)\left(a_{2}^{+}+a_{3}^{+}\right) a_{1}^{-}\right. \\
&\left.\quad+\left(\tilde{N}_{1} \tilde{N}_{2}\left(\rho_{A}-\rho_{B}\right)+\tilde{N}_{2} \tilde{N}_{3} \rho_{A}\right)\left(a_{1}^{+}+a_{3}^{+}\right) a_{2}^{-}\right] .
\end{aligned}
$$

These are the real-time analogues of the spectral representations for the vertex function given by Evans in the imaginary time formalism (ITF) [6]. Explicit expressions for the spectral densities in the "hard thermal loop approximation" were given in Ref. [7] for the 
ITF vertex; their transcription into the real time formalism will be an interesting subject for a separate publication.

\section{B. Relationships between the three-point functions}

From the spectral representation, we obtain relationships between the three-point functions which allow us to eliminate the three $\Gamma_{F}$ 's and $\Gamma_{E}$ in terms of the retarded vertex functions and their complex conjugates. We use again shorthands $N_{i}=N\left(E_{i}\right)=1+2 n\left(E_{i}\right)=$ $1+2 n_{i}$ where $E_{1}, E_{2}, E_{3}$ are the energies flowing into the three legs of the vertex. Using

$$
\begin{aligned}
\operatorname{Re} a_{j}^{ \pm} & =\frac{1}{E_{j}-\Omega_{j}}, \\
\operatorname{Im} a_{j}^{ \pm} & =\mp i \pi \delta\left(E_{j}-\Omega_{j}\right),
\end{aligned}
$$

and doing some tedious algebra we obtain

$$
\begin{aligned}
\Gamma_{F} & =N_{1}\left(\Gamma_{R}^{*}-\Gamma_{R o}\right)+N_{3}\left(\Gamma_{R}^{*}-\Gamma_{R i}\right), \\
\Gamma_{F i} & =N_{2}\left(\Gamma_{R i}^{*}-\Gamma_{R o}\right)+N_{3}\left(\Gamma_{R i}^{*}-\Gamma_{R}\right), \\
\Gamma_{F o} & =N_{1}\left(\Gamma_{R o}^{*}-\Gamma_{R}\right)+N_{2}\left(\Gamma_{R o}^{*}-\Gamma_{R i}\right), \\
\Gamma_{E} & =\Gamma_{R i}^{*}+\Gamma_{R}^{*}+\Gamma_{R o}^{*}+N_{2} N_{3}\left(\Gamma_{R i}+\Gamma_{R i}^{*}\right) \\
& +N_{1} N_{3}\left(\Gamma_{R}+\Gamma_{R}^{*}\right)+N_{1} N_{2}\left(\Gamma_{R o}+\Gamma_{R o}^{*}\right),
\end{aligned}
$$

where we have used the identity

$$
N_{1} N_{2}+N_{2} N_{3}+N_{3} N_{1}=-1 .
$$

Substituting Eq. (33) into Eq. (24) we obtain the analogue of Eq. (12):

$$
\begin{aligned}
\Gamma & =\Gamma_{R}\left(\begin{array}{c}
n_{1} \\
1+n_{1}
\end{array}\right)\left(\begin{array}{l}
1 \\
1
\end{array}\right)\left(\begin{array}{c}
n_{3} \\
1+n_{3}
\end{array}\right)-\frac{1}{2} \Gamma_{R}^{*}\left(N_{1}+N_{3}\right)\left(\begin{array}{l}
1 \\
1
\end{array}\right)\left(\begin{array}{c}
n_{2} \\
1+n_{2}
\end{array}\right)\left(\begin{array}{l}
1 \\
1
\end{array}\right) \\
& +\Gamma_{R i}\left(\begin{array}{l}
1 \\
1
\end{array}\right)\left(\begin{array}{c}
n_{2} \\
1+n_{2}
\end{array}\right)\left(\begin{array}{c}
n_{3} \\
1+n_{3}
\end{array}\right)-\frac{1}{2} \Gamma_{R i}^{*}\left(N_{2}+N_{3}\right)\left(\begin{array}{c}
n_{1} \\
1+n_{1}
\end{array}\right)\left(\begin{array}{l}
1 \\
1
\end{array}\right)\left(\begin{array}{l}
1 \\
1
\end{array}\right) \\
& +\Gamma_{R o}\left(\begin{array}{c}
n_{1} \\
1+n_{1}
\end{array}\right)\left(\begin{array}{c}
n_{2} \\
1+n_{2}
\end{array}\right)\left(\begin{array}{l}
1 \\
1
\end{array}\right)-\frac{1}{2} \Gamma_{R o}^{*}\left(N_{1}+N_{2}\right)\left(\begin{array}{l}
1 \\
1
\end{array}\right)\left(\begin{array}{c}
1 \\
1
\end{array}\right)\left(\begin{array}{c}
n_{3} \\
1+n_{3}
\end{array}\right) .
\end{aligned}
$$


This decomposition, together with the spectral representations given by the first three lines in Eq. (31) which express the three (complex) retarded vertices in terms of two (real) spectral densities, is the main result of this paper. It is mathematically equivalent to, but structurally simpler than the results obtained by Evans [8], Kobes [9], Aurenche and Becherrawy [10], and van Eijck, Kobes, and van Weert [11, [2]. The focus of those papers was the comparison of the imaginary-time and real-time formalisms, and transformations between various representations of the real-time formulation. Relations were obtained between the individual components of the vertex tensor and the retarded and advanced vertices. The basic procedure in all cases was similar: One started with the observation that the propagator can be diagonalized by a simple matrix transformation. This diagonalization procedure is not unique, resulting in different formulations using different basis functions (retarded/advanced [10], $F / \bar{F}$ [12], etc., see [17] for a recent review). One then attempts to write the vertex tensor in a similar way, as a simple core function contracted with a similar matrix transformation at each leg $[8]$. Unfortunately, none of the previously obtained results is particularly simple.

The key to our derivation is the realization that the transformations between the various (anti)symmetric vertices Eq. (22) are much simpler than transformations involving the individual components of the vertex tensor $\Gamma_{a b c}$. In this sense, the (anti)symmetric combinations that we use are more physical than, e.g., the time-ordered vertex $\Gamma_{111}$. Our result Eq. (35), expressed in the column vector notation of [16], is extremely simple to use in calculations, as will be demonstrated in the next section.

\section{OUTLINE OF A CALCULATION WITH FULL VERTICES}

As an example of the usefulness of these techniques, in particular the decomposition Eq. (35), we set up the calculation of the polarization tensor in $\phi^{3}$ theory in six dimensions. This renormalizable theory is interesting as a toy model since the $\phi^{3}$ interaction is mathematically similar to the three gluon interaction in QCD. The Lagrangian is given by 


$$
\mathcal{L}=\frac{1}{2}\left(\partial_{\mu} \phi\right)^{2}-\frac{1}{2} m^{2} \phi^{2}-\frac{g}{3 !} \phi^{3}
$$

In terms of full propagators and vertices the polarization tensor is given by the 1-loop diagram shown in Fig. 1.

In order for the polarization tensor and vertex to satisfy the coupled Schwinger-Dyson equations, one must use one corrected vertex and one bare vertex. We follow the notation of [16]. The bare vertex contributes a factor of $-i g \tau^{3}$, where $\tau^{3}$ is the third Pauli matrix. We obtain

$$
\Pi_{a b}(k)=g \int \frac{d^{6} p}{(2 \pi)^{6}} \tilde{\Gamma}_{c a d}(p-k / 2, k,-(p+k / 2)) D_{b d}(-(p+k / 2)) \tau_{b x}^{3} D_{x c}(p-k / 2)
$$

where $\tilde{\Gamma}$ indicates the full $1 \mathrm{PI}$ vertex. We rewrite this equation in terms of the connected vertex $\Gamma$ by using the definition

$$
\tilde{\Gamma}_{c a d}\left(p_{1}, p_{2}, p_{3}\right)=\frac{1}{i^{3}} D_{c c^{\prime}}^{-1}\left(p_{1}\right) D_{a a^{\prime}}^{-1}\left(p_{2}\right) D_{d d^{\prime}}^{-1}\left(p_{3}\right) \Gamma_{c^{\prime} a^{\prime} d^{\prime}}\left(p_{1}, p_{2}, p_{3}\right)
$$

which gives

$$
\Pi_{a b}(k)=i g D_{a a^{\prime}}^{-1}(k) \tau_{b x}^{3}\left(\int \frac{d^{6} p}{(2 \pi)^{6}} \Gamma_{x a^{\prime} b}(p-k / 2, k,-(p+k / 2))\right) .
$$

We insert the decompositions Eqs. (13) and (35) in terms of outer products of 2component column vectors and use the rule [16] that for internal indices $\left(a^{\prime}, c^{\prime}, d^{\prime}, c, d, x\right)$ which are to be summed over the corresponding two column vectors in Eqs. (13) and (35) must be contracted to a scalar:

$$
\left(\begin{array}{l}
x_{1} \\
x_{2}
\end{array}\right) \cdot\left(\begin{array}{l}
x_{3} \\
x_{4}
\end{array}\right)=x_{1} x_{3}+x_{2} x_{4}
$$

The index $b$ in Eq. (39) is not summed over; the rule [16] is that, after multiplying the $\tau_{3}$-matrix into the vertex by summation over the index $x$, the remaining two column vectors with the index $b$ are to be contracted to a new column vector according to

$$
\left(\begin{array}{l}
x_{1} \\
x_{2}
\end{array}\right)\left(\begin{array}{l}
x_{3} \\
x_{4}
\end{array}\right)=\left(\begin{array}{l}
x_{1} x_{3} \\
x_{2} x_{4}
\end{array}\right)
$$


Since Eq. (35) contains 6 terms and Eq. (13) contains 2 terms, one expects 12 separate terms in the polarization tensor each of which is again a sum of two terms according to Eq. (40). It is, however, known from practical experience that many of these terms cancel. In our formulation this cancellation occurs at the very first step of the calculation because many of the contractions Eq. (40) vanish identically, e.g.

$$
\left(\begin{array}{l}
1 \\
1
\end{array}\right) \cdot\left(\begin{array}{c}
1 \\
-1
\end{array}\right)=0, \quad\left(\begin{array}{c}
1+n \\
-n
\end{array}\right) \cdot\left(\begin{array}{c}
n \\
1+n
\end{array}\right)=0 .
$$

Furthermore, when calculating, for example, the retarded polarization operator, all terms vanish in which the column vector carrying the index $b$ is given by $\left(\begin{array}{c}1 \\ -1\end{array}\right)$.

One thus obtains directly the following simple expressions:

$$
\begin{aligned}
\Pi_{R}(k) & =\Pi_{11}(k)+\Pi_{12}(k) \\
& =i g \frac{1}{D_{R}(k)} \int \frac{d^{6} p}{(2 \pi)^{6}} N_{1}\left(\Gamma_{R o}^{*}-\Gamma_{R}\right) \\
\Pi_{A}(k) & =\Pi_{11}(k)+\Pi_{21}(k) \\
& =i g \frac{1}{D_{A}(k)} \int \frac{d^{6} p}{(2 \pi)^{6}} N_{1}\left(\Gamma_{R}^{*}-\Gamma_{R o}\right)=\Pi_{R}^{*}(k) \\
\Pi_{F}(k) & =\Pi_{11}(k)+\Pi_{22}(k) \\
& =i g \int \frac{d^{6} p}{(2 \pi)^{6}} N_{1} N_{2}\left[\frac{1}{D_{R}(k)}\left(\Gamma_{R o}^{*}-\Gamma_{R}\right)-\frac{1}{D_{A}(k)}\left(\Gamma_{R}^{*}-\Gamma_{R o}\right)\right] .
\end{aligned}
$$

The only additional input were the relations Eqs. (16) and (18) which result in the simple identities

$$
\begin{aligned}
\int \frac{d^{6} p}{(2 \pi)^{6}}\left(\Gamma_{R i}+\Gamma_{R o}\right) & =0 \\
\int \frac{d^{6} p}{(2 \pi)^{6}}\left(\Gamma_{R}+\Gamma_{R}^{*}\right) & =0 .
\end{aligned}
$$

The above results express the components of the full scalar self energy in terms of the full propagators and vertices and are thus correct to all orders of perturbation theory. Formally, the expressions in Eq. (43) and the identities Eq. (44) remain unchanged in $D$ space-time dimensions (with arbitrary $D$ ). The simple structure given in Eq. (43) should be preserved in partially resummed approximation schemes like e.g. the hard thermal loop approximation, in order to remain consistent with the Schwinger-Dyson equations. 


\section{CONCLUSIONS}

We have given a simple decomposition of the eight component real time vertex tensor in terms of seven vertex functions and outer products of triplets of simple two-component column vectors. We have shown that the seven vertex functions can be obtained through a spectral representation from two independent real spectral functions. We have obtained a set of relationships between the seven vertex functions which allows us to express all of them through the three retarded vertex functions and their complex conjugates. These expressions were used to derive another vector decomposition of the vertex tensor in terms of the retarded vertex functions and their complex conjugates. The advantage of this expression for performing calculations is that it allows one to see immediately that, after performing all of the contractions, many of the terms give zero. As far as we can see, our formalism implements all of the known cancellations between contributions from different components

of the propagator and vertex tensors algebraically through the contraction rules for the column vectors. These cancellations occur before any loop integrals must be evaluated, at the very beginning of the calculation. This greatly simplifies loop calculations in real-time finite temperature field theory.

We note that work similar to that presented here was recently done by Fauser and Henning for the Yukawa theory [18].

\section{ACKNOWLEDGMENTS}

M.E.C. gratefully acknowledges support by the Alexander von Humboldt Foundation through a Research Fellowship. U.H. would like to thank B. Müller and the Physics Department at Duke University, were a large fraction of his work was done during a sabbatical leave, for their warm hospitality and stimulating discussions. The work of U.H. was supported by DFG, BMBF, and GSI. 


\section{APPENDIX A: LEHMANN SPECTRAL REPRESENTATIONS}

\section{Single-particle propagators}

We give a short derivation of Eq. (7). We start from Eqs. (5) and (6) and evaluate the thermal expectation value in the Heisenberg picture by inserting complete sets of energy and momentum eigenstates. Exploiting translation invariance in the form

$$
\phi(x)=e^{i p \cdot x} \phi(0) e^{-i p \cdot x}
$$

where $p$ is the momentum (gradient) operator and $\phi(0)$ the Schrödinger field operator, we get

$$
\begin{aligned}
\left\langle[\phi(x) \phi(y)]_{ \pm}\right\rangle & =\operatorname{Tr}\left(e^{-\beta H}[\phi(x) \phi(y)]_{ \pm}\right) \\
& =\sum_{m, n} e^{-\beta E_{m}}|\langle m|\phi(0)| n\rangle|^{2}\left(e^{i\left(p_{m}-p_{n}\right) \cdot(x-y)} \pm e^{i\left(p_{m}-p_{n}\right) \cdot(y-x)}\right)
\end{aligned}
$$

where $p_{m, n}^{0}=E_{m, n}$. Fourier transforming with respect to $x-y$ one finds

$$
\begin{aligned}
\rho_{ \pm}(p) & \equiv \int d^{4}(x-y) e^{i p(x-y)}\left\langle[\phi(x), \phi(y)]_{ \pm}\right\rangle \\
& =(2 \pi)^{4} \sum_{m, n}\left|\phi_{m n}(0)\right|^{2} \delta\left(p+p_{m}-p_{n}\right)\left(e^{-\beta E_{m}} \pm e^{-\beta E_{n}}\right) .
\end{aligned}
$$

Using the energy conserving $\delta$-function we can write

$$
e^{-\beta E_{m}}+e^{-\beta E_{n}}=\left(1+2 n\left(p_{0}\right)\right)\left(e^{-\beta E_{m}}-e^{-\beta E_{n}}\right) .
$$

From Eqs. (A3), (A4), (5) and (6) we obtain

$$
\begin{aligned}
D_{F}(p) & =-i \rho_{+}(p)=-i\left(1+2 n\left(p_{0}\right)\right) \rho_{-}(p) \\
& =\left(1+2 n\left(p_{0}\right)\right)\left(D_{R}(p)-D_{A}(p)\right)
\end{aligned}
$$

in agreement with Eq. (7).

Alternatively, one can start from the KMS condition [19]

$$
\left\langle A\left(\mathbf{x}, x_{0}\right) B\left(\mathbf{y}, y_{0}\right)\right\rangle=\left\langle B\left(\mathbf{y}, y_{0}-i \beta\right) A\left(\mathbf{x}, x_{0}\right)\right\rangle=\left\langle B\left(\mathbf{y}, y_{0}\right) A\left(\mathbf{x}, x_{0}+i \beta\right)\right\rangle
$$


and apply it to $\langle\phi(x) \phi(y) \pm \phi(y) \phi(x)\rangle$. In momentum space this leads directly to

$$
\rho_{+}(p)=\left(1+2 n\left(p_{0}\right)\right) \rho_{-}(p)
$$

where $\rho_{ \pm}$are the respective Fourier transforms, with Lehmann representations Eq. (A3).

\section{Three point functions}

In this section we show in more detail how to obtain the spectral representation for the seven connected vertex functions. We will show that the seven vertex functions can be written in terms of two independent spectral functions. We define

$$
\begin{aligned}
& a_{1}=\phi_{1} \phi_{2} \phi_{3} \theta_{12} \theta_{23}, \\
& b_{1}=\phi_{3} \phi_{1} \phi_{2} \theta_{12} \theta_{23}, \\
& c_{1}=\phi_{3} \phi_{2} \phi_{1} \theta_{12} \theta_{23}, \\
& d_{1}=\phi_{2} \phi_{1} \phi_{3} \theta_{12} \theta_{23} .
\end{aligned}
$$

We have similar definitions for other combinations of theta functions. Using an obvious short hand notation we have

$$
\begin{aligned}
& \{a, b, c, d\}_{2}=\left\{\phi_{1} \phi_{2} \phi_{3}, \phi_{2} \phi_{3} \phi_{1}, \phi_{3} \phi_{2} \phi_{1}, \phi_{1} \phi_{3} \phi_{2}\right\} \theta_{32} \theta_{21} \\
& \{a, b, c, d\}_{3}=\left\{\phi_{1} \phi_{2} \phi_{3}, \phi_{2} \phi_{3} \phi_{1}, \phi_{3} \phi_{2} \phi_{1}, \phi_{1} \phi_{3} \phi_{2}\right\} \theta_{23} \theta_{31} \\
& \{a, b, c, d\}_{4}=\left\{\phi_{1} \phi_{2} \phi_{3}, \phi_{3} \phi_{1} \phi_{2}, \phi_{3} \phi_{2} \phi_{1}, \phi_{2} \phi_{1} \phi_{3}\right\} \theta_{21} \theta_{13} \\
& \{a, b, c, d\}_{5}=\left\{\phi_{2} \phi_{3} \phi_{1}, \phi_{3} \phi_{1} \phi_{2}, \phi_{1} \phi_{3} \phi_{2}, \phi_{2} \phi_{1} \phi_{3}\right\} \theta_{13} \theta_{32} \\
& \{a, b, c, d\}_{6}=\left\{\phi_{2} \phi_{3} \phi_{1}, \phi_{3} \phi_{1} \phi_{2}, \phi_{1} \phi_{3} \phi_{2}, \phi_{2} \phi_{1} \phi_{3}\right\} \theta_{31} \theta_{12}
\end{aligned}
$$

In terms of these definitions, the seven vertex functions are

$$
\begin{gathered}
\Gamma_{R}=-\left(a_{3}-b_{3}+c_{3}-d_{3}\right)-\left(a_{4}-b_{4}+c_{4}-d_{4}\right), \\
\Gamma_{R i}=\left(a_{1}-b_{1}+c_{1}-d_{1}\right)+\left(a_{5}-b_{5}+c_{5}-d_{5}\right), \\
\Gamma_{R o}=\left(a_{2}-b_{2}+c_{2}-d_{2}\right)-\left(a_{6}-b_{6}+c_{6}-d_{6}\right),
\end{gathered}
$$




$$
\begin{aligned}
\Gamma_{F}= & \left(a_{1}+b_{1}-c_{1}-d_{1}\right)-\left(a_{2}+b_{2}-c_{2}-d_{2}\right)-\left(a_{5}-b_{5}-c_{5}+d_{5}\right)-\left(a_{6}-b_{6}-c_{6}+d_{6}\right), \\
\Gamma_{F i}= & \left(b_{2}-a_{2}+c_{2}-d_{2}\right)-\left(a_{3}-b_{3}-c_{3}+d_{3}\right)-\left(a_{4}+b_{4}-c_{4}-d_{4}\right)+\left(a_{6}+b_{6}-c_{6}-d_{6}\right), \\
\Gamma_{F o}= & \left(a_{1}-b_{1}-c_{1}+d_{1}\right)+\left(a_{3}+b_{3}-c_{3}-d_{3}\right)+\left(a_{4}-b_{4}-c_{4}+d_{4}\right)-\left(a_{5}+b_{5}-c_{5}-d_{5}\right), \\
\Gamma_{E}= & \left(a_{1}+b_{1}+c_{1}+d_{1}\right)+\left(a_{2}+b_{2}+c_{2}+d_{2}\right)+\left(a_{3}+b_{3}+c_{3}+d_{3}\right)+\left(a_{4}+b_{4}+c_{4}+d_{4}\right) \\
& +\left(a_{5}+b_{5}+c_{5}+d_{5}\right)+\left(a_{6}+b_{6}+c_{6}+d_{6}\right) .
\end{aligned}
$$

We calculate the expectation values of the form Eq. (25) by using Eq. (A1), inserting complete sets of states between the operators and using the cyclic property of the trace. We obtain the four spectral functions Eq. (27).

Using the notation of Eq. (30), the 24 functions Eqs. (A7) and ( terms of the four spectral functions (27) as

$$
\begin{gathered}
\left\{\begin{array}{c}
\left(a_{1}-b_{1}\right) \\
\left(a_{1}+b_{1}\right) \\
\left(c_{1}-d_{1}\right) \\
-\left(c_{1}+d_{1}\right)
\end{array}\right\}=\int_{-\infty}^{\infty} \frac{d \omega_{1}}{2 \pi} \frac{d \omega_{2}}{2 \pi} a_{1}^{+} a_{3}^{-}\left\{\begin{array}{c}
\rho_{1} \\
-\tilde{N}_{3} \rho_{1} \\
\rho_{3} \\
-\tilde{N}_{3} \rho_{3}
\end{array}\right\}, \\
\left\{\begin{array}{c}
\left(a_{2}-b_{2}\right) \\
\left(a_{2}+b_{2}\right) \\
\left(c_{2}-d_{2}\right) \\
-\left(c_{2}+d_{2}\right)
\end{array}\right\}=\int_{-\infty}^{\infty} \frac{d \omega_{1}}{2 \pi} \frac{d \omega_{2}}{2 \pi} a_{1}^{-} a_{3}^{+}\left\{\begin{array}{c}
\rho_{1}-\rho_{2} \\
\tilde{N}_{1}\left(\rho_{1}-\rho_{2}\right) \\
\rho_{3}-\rho_{4} \\
\tilde{N}_{1}\left(\rho_{3}-\rho_{4}\right)
\end{array}\right\}, \\
\left\{\begin{array}{c}
\left(a_{3}-b_{3}\right) \\
\left(a_{3}+b_{3}\right) \\
\left(c_{3}-d_{3}\right) \\
-\left(c_{3}+d_{3}\right)
\end{array}\right\}=\int_{-\infty}^{\infty} \frac{d \omega_{1}}{2 \pi} \frac{d \omega_{2}}{2 \pi} a_{1}^{-} a_{2}^{+}\left\{\begin{array}{c}
\rho_{1}-\rho_{2} \\
\tilde{N}_{1}\left(\rho_{1}-\rho_{2}\right) \\
\rho_{3}-\rho_{4} \\
\tilde{N}_{1}\left(\rho_{3}-\rho_{4}\right)
\end{array}\right\},
\end{gathered}
$$




$$
\begin{gathered}
\left\{\begin{array}{c}
\left(a_{4}-b_{4}\right) \\
\left(a_{4}+b_{4}\right) \\
\left(c_{4}-d_{4}\right) \\
-\left(c_{4}+d_{4}\right)
\end{array}\right\}=\int_{-\infty}^{\infty} \frac{d \omega_{1}}{2 \pi} \frac{d \omega_{2}}{2 \pi} a_{2}^{+} a_{3}^{-}\left\{\begin{array}{c}
\rho_{1} \\
-\tilde{N}_{3} \rho_{1} \\
\rho_{3} \\
-\tilde{N}_{3} \rho_{3}
\end{array}\right\}, \\
\left\{\begin{array}{c}
\left(a_{5}-b_{5}\right) \\
\left(a_{5}+b_{5}\right) \\
\left(c_{5}-d_{5}\right) \\
-\left(c_{5}+d_{5}\right)
\end{array}\right\}=\int_{-\infty}^{\infty} \frac{d \omega_{1}}{2 \pi} \frac{d \omega_{2}}{2 \pi} a_{1}^{+} a_{2}^{-}\left\{\begin{array}{c}
\rho_{2} \\
-\tilde{N}_{2} \rho_{2} \\
\rho_{4} \\
-\tilde{N}_{2} \rho_{4}
\end{array}\right\}, \\
\left\{\begin{array}{c}
\left(a_{6}-b_{6}\right) \\
\left(a_{6}+b_{6}\right) \\
\left(c_{6}-d_{6}\right) \\
-\left(c_{6}+d_{6}\right)
\end{array}\right\}=\int_{-\infty}^{\infty} \frac{d \omega_{1}}{2 \pi} \frac{d \omega_{2}}{2 \pi} a_{2}^{-} a_{3}^{+}\left\{\begin{array}{c}
\rho_{2} \\
-\tilde{N}_{2} \rho_{2} \\
\rho_{4} \\
-\tilde{N}_{2} \rho_{4}
\end{array}\right\} .
\end{gathered}
$$

Not all of the spectral functions Eq. (27) are independent. We can define the two independent combinations Eq. (26) and express all results in terms of $\rho_{A}$ and $\rho_{B}$ by using the relations

$$
\begin{gathered}
\rho_{1}-\rho_{3}=\tilde{N}_{1}\left(\rho_{A}-\rho_{B}\right)-\tilde{N}_{2} \rho_{B}, \\
\rho_{2}-\rho_{4}=\tilde{N}_{1}\left(\rho_{A}-\rho_{B}\right)+\tilde{N}_{3} \rho_{A} .
\end{gathered}
$$

Using Eqs. (A9), (A10) and (A11) we obtain Eq. (31) for the spectral representation of the vertex functions. 


\section{REFERENCES}

$\dagger \quad$ Humboldt Fellow. Permanent address: Department of Physics and Winnipeg Institute of Theoretical Physics, University of Winnipeg, Winnipeg, Manitoba, Canada R3B 2E9.

[1] L.V. Keldysh, Zh. Eksp. Teor. Fiz. 47, 1515 (1964).

[2] E.M. Lifshitz and L.P. Pitaevski, Physical Kinetics (Pergamon Press, Oxford, 1981).

[3] J. Rammer and H. Smith, Rev. Mod. Phys. 58, 323 (1986).

[4] V.V. Lebedev and A.V. Smilga, Physica A 181, 187 (1992).

[5] M.E. Carrington, Phys. Rev. D48, 3836 (1993).

[6] T.S. Evans, Phys. Lett. B249, 286 (1990).

[7] J.C. Taylor, Phys. Rev. D48, 958 (1993).

[8] T.S. Evans, Phys. Lett. B252, 108 (1990); and Nucl. Phys. B374, 340 (1992).

[9] R. Kobes, Phys. Rev. Lett., 67, 1384 (1991); and Phys. Rev. D43, 1269 (1991).

[10] P. Aurenche and T. Becherrawy, Nucl. Phys. B379, 259 (1992).

[11] M.A. van Eijck and Ch.G. van Weert, Phys. Lett. B278, 305 (1992).

[12] M.A. van Eijck, R. Kobes, and Ch.G. van Weert, Phys. Rev. D50, 4097 (1994).

[13] N. Landsman and Ch.G. van Weert, Phys. Rep. 145, 141 (1987).

[14] H.B. Callen and T.A. Welton, Phys. Rev. 83, 34 (1951).

[15] K.-C. Chou, Z.-B. Su, B.-L. Hao and L. Yu, Phys. Rep. 118, 1 (1985).

[16] P.A. Henning, Phys. Rep. 253, 235 (1995).

[17] F. Gelis, Los Alamos eprint archive hep-ph/9701410.

[18] R. Fauser and P.A. Henning, private communication. 
[19] R. Kubo, J. Phys. Soc. Japan 12, 570 (1957); and Rep. Progr. Phys. 29, 255 (1966); P.C. Martin and J. Schwinger, Phys. Rev 115, 1432 (1959). 


\section{FIGURES}

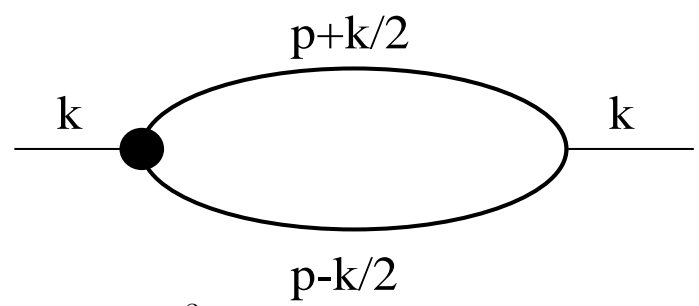

FIG. 1. The scalar self energy in $\phi^{3}$ theory. The thick lines represent full propagators and the solid blob represents a full three-point vertex. 